\title{
Minimum number of spermatozoa required for normal fertility after deep intrauterine insemination in non-sedated sows
}

\author{
E. A. Martinez ${ }^{1}$, J. M. Vazquez ${ }^{1}$, J. Roca ${ }^{1}$, X. Lucas ${ }^{1}$, M. A. Gil ${ }^{1}$, \\ I. Parrilla ${ }^{1}$, J. L. Vazquez ${ }^{2}$ and B. N. Day ${ }^{3}$ \\ ${ }^{1}$ Department of Animal Pathology, University of Murcia, 30071 Murcia, Spain; ${ }^{2}$ Department \\ of Surgery, University Miguel Hernandez, Elche, Spain; and ${ }^{3}$ Department of Animal \\ Sciences, University of Missouri-Columbia, Columbia, MO 65211, USA
}

\begin{abstract}
A fibreoptic endoscope procedure for non-surgical deep intrauterine insemination in non-sedated sows has been reported. However, the endoscope is an expensive and fragile instrument, and is unsuitable for use under field conditions. The aim of this study was to determine the minimum number of spermatozoa required to maintain optimal fertility using a flexible catheter $(1.8 \mathrm{~m}$ in length, $4 \mathrm{~mm}$ in diameter) for deep intrauterine insemination in 2-6 parity non-sedated sows. Crossbred sows were treated with eCG $24 \mathrm{~h}$ after weaning and with hCG $72 \mathrm{~h}$ later to induce oestrus. Deep intrauterine insemination was performed $36 \mathrm{~h}$ after hCG treatment in 117, 126, 60 and 69 sows with $15.0,5.0,2.5$ or $1.0 \times 10^{7}$ spermatozoa in $10 \mathrm{ml}$, respectively. Weaned sows $(n=147)$ not treated with hormones and used for standard artificial insemination (AI) (two inseminations per oestrus with $3 \times 10^{9}$ spermatozoa in $100 \mathrm{ml}$ ) served as controls. The flexible catheter was passed successfully through the cervix into one uterine horn in $95.4 \%$ of the sows in an average of
\end{abstract}

\section{Introduction}

Artificial insemination (AI) is of great importance in the pig industry. Over the past decade, there has been an increase in the development of field $\mathrm{Al}$ services in many countries. In 29 countries in which data are available, 24.1 million sows are currently inseminated artificially $(48 \%$ of the total number of sows), and in some countries, the percentage of sows inseminated artificially is greater than $80 \%$ (Weitze, 2000). The development of a technique that would allow a more efficient use of semen samples from genetically superior boars would be of great value to the pig industry and would complement the development of Al. Important advances are being made in the development of new aspects of the $\mathrm{Al}$ technique (for example, improvement of semen extenders, tests to evaluate the quality, functionality and fertilizing ability of spermatozoa, strategies to determine the time of ovulation). However, current requirements of volume and sperm dosage to obtain optimal fertility using

Email:emilio@um.es
$3.7 \pm \mathbf{0 . 0 9} \mathbf{m i n}$. Farrowing rates after deep intrauterine insemination with 15 or $5 \times 10^{7}$ spermatozoa did not differ from those of the control group (82.9, 76.2 and $83.0 \%$, respectively), but a significant decrease $(P<0.001)$ was observed in sows inseminated with 2.5 or $1.0 \times 10^{7}$ spermatozoa (46.7 and $39.1 \%$, respectively). In contrast, the number of spermatozoa inseminated did not affect prolificacy. Laparotomy revealed that the tip of the flexible catheter reached approximately the anterior third of the uterine horn. Although deep intrauterine insemination was performed in only one uterine horn, the percentages of embryos collected from the tip of both uterine horns 2 days after deep insemination were not significantly different. The results show that in comparison with standard Al, a 20-60-fold reduction in the number of spermatozoa inseminated and an 8-10-fold reduction in the dose volume can be achieved without decreasing fertility when semen is deposited non-surgically into the upper first third of one uterine horn. the standard technique of intracervical Al $\left(2.5-4.0 \times 10^{9}\right.$ spermatozoa per insemination in $80-100 \mathrm{ml}$ of liquid) are similar to those recommended at the inception of pig Al (Wiggins et al., 1951; Polge, 1956; Dziuk and Henshaw, 1958; Stratman and Self, 1968; Baker et al., 1968). This large number of spermatozoa limits the number of doses that can be prepared from one semen sample to approximately 20-25.

However, it is now known that the number of spermatozoa per insemination can be reduced markedly if the sperm dose is deposited into the uterine horn instead of into the cervix. In pigs, a 100-fold reduction of the standard pig Al dose $\left(3 \times 10^{9}\right.$ spermatozoa in $80 \mathrm{ml}$ fluid) can be made when spermatozoa are deposited surgically close to the uterotubal junction (Krueger et al., 1999; Krueger and Rath, 2000). Despite the complex anatomy of the cervix and uterine horn of the sow, a new fibreoptic endoscope procedure for successful non-surgical deep intrauterine insemination in non-sedated sows has been reported (Vazquez et al., 1999). Endoscopic non-surgical deep intrauterine insemination can be performed quickly in sows, and preliminary 


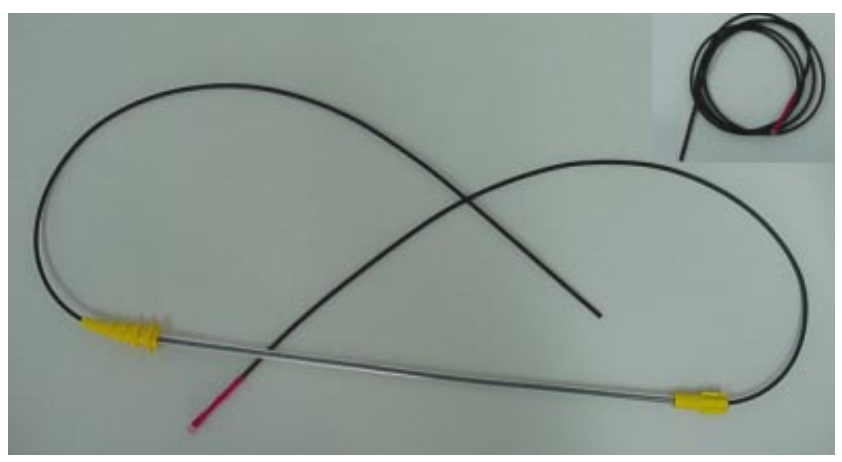

Fig. 1. Catheter used for non-surgical deep intrauterine embryo transfer in the sow. The flexibility of the catheter can be observed in the inset.

results indicate that normal farrowing rates and litter sizes are obtained after inseminating only $5 \times 10^{7}$ spermatozoa per sow in $10 \mathrm{ml}$ (Martinez et al., 2000). However, although endoscopic deep intrauterine insemination is successful for inseminating sows with a small number of spermatozoa, the endoscope is expensive and fragile, and is unsuitable for use under field conditions.

The aim of this study was to evaluate the effectiveness of a new flexible catheter to gain access to the uterine horn through the cervix and to determine the minimum number of spermatozoa required to maintain optimal fertility after deep intrauterine insemination in non-sedated sows.

\section{Materials and Methods}

\section{Animals}

Experiments were conducted under field conditions in a commercial pig farm in Murcia (Spain). For 23 weeks, crossbred sows were selected each week on the day of weaning. Sows were allocated individually to crates in a mechanically ventilated confinement facility and fed with a commercial ration twice a day. Water was provided ad libitum for $2 \mathrm{~h}$ after feeding.

\section{Induction and detection of oestrus}

Oestrus was induced in each female by an i.m. injection of 1250 iu equine chorionic gonadotrophin (eCG) (Folligon; Intervet International BV, Boxmeer) $24 \mathrm{~h}$ after weaning followed by administration of 750 iu hCG (Chorulon; Intervet International BV) $72 \mathrm{~h}$ later. Oestrous detection was performed once each day (07:00 h), beginning 2 days after the eCG injection, by allowing females nose to nose contact with a mature boar and by applying back pressure. Sows that showed a standing heat reflex were considered to be in oestrus and were used for deep intrauterine insemination if oestrus was detected within $24 \mathrm{~h}$ after the hCG injection.

\section{Deep intrauterine inseminations}

Three sexually mature hybrid boars of proven fertility and with satisfactory semen characteristics in the 10 weeks preceding the experiment were selected as semen donors. Sperm-rich fractions were collected once a week from the three boars using the gloved-hand method, and concentration, motility and morphology of spermatozoa were determined. Immediately after evaluation, semen was diluted to $3 \times 10^{7}$ cells $\mathrm{ml}^{-1}$ in Beltsville thawing solution (BTS) diluent (Pursel and Johnson, 1975) at $35^{\circ} \mathrm{C}$. The sperm suspensions were maintained for up to $24 \mathrm{~h}$ at $17^{\circ} \mathrm{C}$. Immediately before the inseminations, sperm motility was examined again and semen samples were resuspended, if necessary, in BTS diluent at $17^{\circ} \mathrm{C}$ to provide homospermic doses of 15.0, 5.0, 2.5 or $1.0 \times 10^{7}$ spermatozoa in $5 \mathrm{ml}$ for deep intrauterine insemination (experimental groups). A total of 413 sows (2-6 parity) were weaned at day $21.23 \pm 0.09$, and treated with eCG $24 \mathrm{~h}$ after weaning and with hCG $72 \mathrm{~h}$ later to induce oestrus, and then assigned to the experimental groups. Deep intrauterine insemination was performed once in one uterine horn $36 \mathrm{~h}$ after hCG treatment. Non-hormonally treated sows $(n=147$; duration of lactation $21.00 \pm 0.14$ days; $2-6$ parity) from the same batch of weaned sows as the experimental sows and that showed the onset of oestrus 4 days after weaning were used for standard $\mathrm{Al}$ (control group). These sows were inseminated twice at 0 and $24 \mathrm{~h}$ after the onset of oestrus with the standard insemination dose $\left(3 \times 10^{9}\right.$ spermatozoa diluted to $100 \mathrm{ml}$ in BTS diluent at $30^{\circ} \mathrm{C}$ ) prepared from the same semen samples that were used for the experimental group. Sows from experimental and control groups were not provided with the presence of a boar during the insemination.

Deep uterine inseminations were performed in each sow in gestation crates, without sedation. After thorough cleaning of the perineal area of the sows, a commercial Al spirette (Minitub, Tiefenbach) was inserted through the vagina into the cervix and was used to manipulate a specially designed flexible catheter (working length $1.80 \mathrm{~m}$, outer diameter 4 $\mathrm{mm}$, diameter of the inner tubing $1.80 \mathrm{~mm}$ ) (Fig. 1). The flexible catheter was inserted through the spirette, passed through the cervical canal and moved forward along one uterine horn until its total length had been inserted. Before inseminations were performed, the inner tubing of the flexible catheter was rinsed with BTS diluent and refilled with approximately $2 \mathrm{ml}$ of BTS extender at $30^{\circ} \mathrm{C}$. Predetermined insemination doses of spermatozoa in $5 \mathrm{ml}$ at $30^{\circ} \mathrm{C}$ were flushed into one uterine horn using a $5 \mathrm{ml}$ disposable syringe attached to the inner tubing of the flexible catheter. Subsequently, an additional $5 \mathrm{ml}$ of BTS diluent was used to force all remaining spermatozoa out of the flexible catheter. The flexible catheters were cleaned externally and internally with BTS diluent at $35^{\circ} \mathrm{C}$ between insertions.

The behaviour of the sows, the difficulties encountered during the insertion of the flexible catheter and the duration of the procedure were recorded in each case. Pregnancy was diagnosed at days 24-28 after insemination by transcutaneous ultrasonography (Pie Medical, Maastricht). All pregnant sows were allowed to carry litters to term, and farrowing rates and litter sizes were recorded. 


\section{Evaluation of the position of the flexible catheter in the uterine horn}

Six weaned sows (2-3 parity) were examined by laparotomy to evaluate the position of the flexible catheter in the uterine horn. Oestrus was induced as described previously. On the day of hCG administration, sows were moved from the commercial farm to the Veterinary Hospital of the University of Murcia. The sows were housed in gestation crates and the flexible catheter was inserted 34-37 h after hCG treatment. The end of the flexible catheter was fixed to the tail of the sow with tape and the sow was sedated with an i.m. injection of azaperone (3 mg $\mathrm{kg}^{-1}$ ) (Stresnil; Lab. Esteve Veterinaria, Barcelona). General anaesthesia was induced i.v. by a combination of xylazine (1 mg kg-1; Rompum; Bayer, Barcelona) and ketamine (3 mg kg-1; Imalgene 1000; Lab. Rhoie Merieux, Barcelona), and was maintained with halothane (0.7-1.5\%). The reproductive tract was exposed via a mid-line incision, and the position of the flexible catheter and the distance from the tip of the flexible catheter to the uterotubal junction were determined.

\section{Collection of embryos after deep intrauterine insemination}

Six sows (2-6 parity) were inseminated once with $15 \times 10^{7}$ spermatozoa at $36 \mathrm{~h}$ after hCG treatment and subjected to laparotomy on day 4 of the oestrous cycle (day $0=$ onset of oestrus) to evaluate whether only ipsilateral fertilization occurred after deep intrauterine insemination. Anaesthesia was induced as described previously. After exposure of the genital tract, corpora lutea were counted on the ovaries, and both oviducts and the tip of the uterine horns were flushed with Tyrode's albumin lactate pyruvate (TALP)-Hepes medium (Hagen et al., 1991). Embryos and oocytes were collected as described by Day (1979). In brief, after clamping the uterine horn $20-30 \mathrm{~cm}$ below the uterotubal junction with bowl forceps, $20 \mathrm{ml}$ of TALPHepes medium was introduced into the uterine horn via the oviduct. A further $20 \mathrm{ml}$ of TALP-Hepes was injected into the uterine horn on the ovarian side of the forceps after the insertion of a cannula through the uterine wall near the uterotubal junction. Examination of embryos was performed under a stereoscope at a magnification of $\times 60$. One-cell eggs and embryos with abnormal cleavage or morphology were classified as unfertilized oocytes and degenerated embryos, respectively. The remaining embryos were considered normal.

\section{Statistical analysis}

All means are expressed as least squares (Is) \pm SEM. Data were analysed using SAS/STAT (1999) and differences were considered significant at $P<0.05$. Difficulties encountered in inserting the flexible catheter through the cervix were evaluated by three experienced inseminators and were divided into four classes: (i) no or minor difficulties, (ii) medium difficulties, (iii) high difficulties or (iv) impossible, depending on the resistance as assessed by the inseminator during the insertions. The distributions of sows over the three parity classes (2, 3 or 4-6 parity) and over the three periods of lactation (days 16-20, days 21-23 or days 24-27) in the five classes of sperm dosage (four classes for deep intrauterine insemination groups and one class for the control group) were evaluated using the FREQ procedure. The percentage of sows within each difficulty group was compared using the chi-squared test. The CATMOD procedure was used to analyse the following: (i) the effect of the number of parity (three classes: 2, 3 or 4-6 parity), duration of lactation (three classes: days 16-20, days 21-23 or days 23-27) and the interaction of both parameters on the difficulty rating encountered in inserting the flexible catheter; (ii) the influence of the difficulty rating in inserting the flexible catheter and the influence of the AI system used (deep intrauterine or standard inseminations) on the behaviour of the sows during insemination (three classes: good, moderate or bad, depending on the reaction of the sow during insertion of the flexible catheter); and (iii) the effect of the number of spermatozoa inseminated on pregnancy and farrowing rates. The time required to complete the procedure in each difficulty class, the effect of the number of parity (three classes: 2, 3 or 4-6 parity), the duration of lactation (three classes: days 16-20, days 21-23 or days 24-27) and the interaction of both parameters on the duration of the deep intrauterine insemination and the effect of the number of spermatozoa inseminated on the number of live and stillborn piglets, and on litter size were analysed using GLM procedure. When ANOVA revealed a significant effect, values were compared using the Tukey-Kramer adjustment for multiple comparisons. The influence of deep intrauterine insemination on the proportions of normal embryos collected from the ipsilateral and contralateral uterine horns was evaluated using the GLM procedure after arcsin transformation of the proportions of normal embryos. It was not possible to determine in which uterine horn the sperm dose was deposited during deep intrauterine insemination. Therefore, the data with the highest percentages of normal embryos (or the highest number of embryos, when the proportion was the same) collected from each uterine horn of the sows subjected to laparotomy were classified within the same uterine horn to establish the maximum difference possible in the proportion of normal embryos between ipsilateral and contralateral uterine horns.

\section{Results}

In total, 425 sows were treated with hormones to induce oestrus. Twenty-three sows (5.4\%) showed no obvious signs of oestrus at $24 \mathrm{~h}$ after hCG injection and were excluded from the study. Three hundred and ninety sows were used for deep intrauterine insemination and the other 12 sows were subjected to laparotomy.

The difficulties encountered during the insertion of the flexible catheter and the time required to complete the 
Table 1. Pregnancy and farrowing rates, and litter sizes of hormonally treated weaning sows at oestrus after deep intrauterine insemination of different numbers of spermatozoa using a flexible catheter at $36 \mathrm{~h}$ after hCG administration

\begin{tabular}{|c|c|c|c|c|c|c|}
\hline \multirow{2}{*}{$\begin{array}{l}\text { Spermatozoa } \\
\left(\times 10^{7}\right)\end{array}$} & \multirow{2}{*}{$\begin{array}{l}\text { Sows } \\
(n)\end{array}$} & \multirow{2}{*}{$\begin{array}{l}\text { Pregnancy } \\
\text { rate }(\%)^{*}\end{array}$} & \multirow{2}{*}{$\begin{array}{l}\text { Farrowing } \\
\text { rate }(\%)\end{array}$} & \multicolumn{3}{|c|}{$\begin{array}{c}\text { Piglets born } \\
\text { (least squares mean } \pm \text { SEM) }\end{array}$} \\
\hline & & & & Total & Live & Stillborn \\
\hline 1.0 & 69 & $39.1^{\mathrm{a}}$ & $39.1^{a}$ & $9.44 \pm 0.36$ & $9.03 \pm 0.38$ & $0.41 \pm 0.15$ \\
\hline 2.5 & 60 & $51.7^{a}$ & $46.7^{\mathrm{a}}$ & $9.3 \pm 0.35$ & $8.75 \pm 0.37$ & $0.57 \pm 0.15$ \\
\hline 5.0 & 126 & $77.8^{\mathrm{b}}$ & $76.2^{\mathrm{b}}$ & $9.4 \pm 0.19$ & $8.91 \pm 0.20$ & $0.49 \pm 0.08$ \\
\hline 15.0 & 117 & $86.3^{b}$ & $82.9^{b}$ & $9.70 \pm 0.19$ & $9.30 \pm 0.20$ & $0.40 \pm 0.08$ \\
\hline Control $^{+}$ & 147 & $86.4^{\mathrm{b}}$ & $83.0^{b}$ & $9.97 \pm 0.17$ & $9.40 \pm 0.18$ & $0.57 \pm 0.07$ \\
\hline
\end{tabular}

*Pregnancy was diagnosed using ultrasonography at days 24-28 after insemination.

${ }^{+}$Non-hormonally treated sows at oestrus after weaning were inseminated twice intracervically with $3 \times 10^{9}$ spermatozoa in $100 \mathrm{ml}$ of fluid using a traditional Al spirette.

abValues within the same column with different superscripts are significantly different $(P<0.001)$.

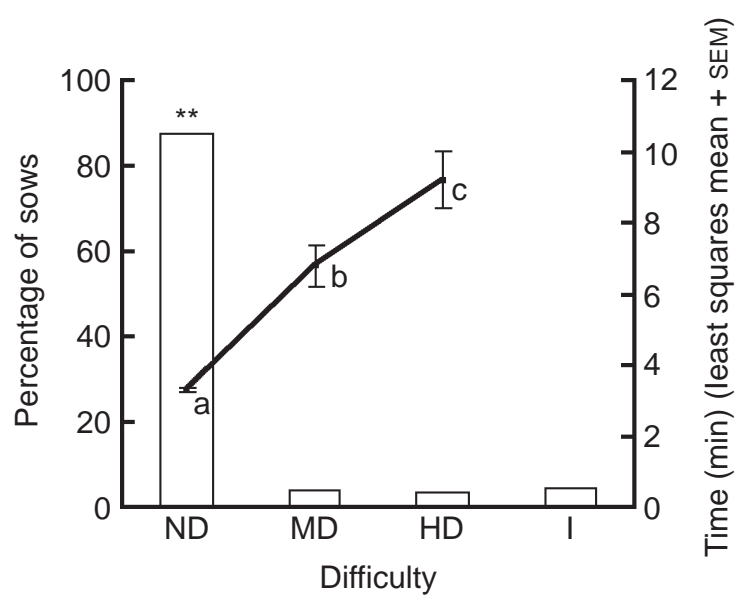

Fig. 2. The difficulties encountered during the insertion of the flexible catheter through the cervical canal into one uterine horn in sows $(n=390)$ and the time required to complete the procedure. Difficulties encountered were divided into four classes: ND: no or minor difficulties, MD: medium difficulties, HD: high difficulties, and I: impossible. ${ }^{* *} P<0.0001$ among bars. abc $P<0.0001$ among points of the line.

procedure are shown (Fig. 2). It was possible to insert the flexible catheter through the cervix into one uterine horn in $95.4 \%$ of sows in an average of $3.71 \pm 0.09 \mathrm{~min}$. The number of the parity (2,3 or 4-6 parities) or the duration of lactation (days $16-20$, days $21-23$ or days $24-27$ ), or the interaction of both factors had no significant effect on the difficulties encountered when inserting the flexible catheter or on the time required to insert the catheter.

The behaviour of the sows during the procedure was classified as good or moderate in 97.1, 93.8, 85.7 and $94.4 \%$ for no or minor difficulties, medium difficulties, high difficulties and impossible groups, respectively and the differences were not significant. The method of insemination (deep intrauterine insemination and standard $\mathrm{Al}$ ) also had no significant influence on the behaviour of the sows.
The percentage of sows that showed good, moderate and bad reaction during deep intrauterine insemination or standard insemination was $91.5,4.9$ and 3.6, and 86.4, 8.8 and $4.8 \%$, respectively.

In 18 of 390 sows used for deep intrauterine insemination it was not possible to insert the flexible catheter through the cervix and these animals were not included in the insemination trials. Therefore, 372 sows were deeply inseminated in one uterine horn using the flexible catheter (experimental groups) and 147 sows were standard inseminated (control group). The distribution of the percentages of sows from the three classes of parity (2, 3 and 4-6 parity) and from the three classes of duration of lactation (days 16-20, days 21-23 and days 24-27) in the five groups of sperm dosage (four experimental and one control) was not significantly different. Insemination results are shown (Table 1). Thirteen sows detected as pregnant at days 24-28 after insemination failed to farrow due to loss of fetuses or death $(1.6$ and $0.8 \%$, and 2.0 and $0.7 \%$ for experimental and control groups, respectively). In two sows $(0.5 \%)$ from the experimental groups and one sow $(0.7 \%)$ from the control group, vaginal discharges at days 15-29 after insemination were observed. Pregnancy and farrowing rates after deep intrauterine insemination with 15 or $5 \times 10^{7}$ spermatozoa did not differ from those of the control group, but a significant decrease $(P<0.001)$ was observed in sows inseminated with 2.5 or $1 \times 10^{7}$ spermatozoa. In contrast, the number of spermatozoa inseminated did not significantly affect the mean number of live or stillborn pigs, or the total number of piglets born.

Surgical observations in six sows revealed that the tip of the flexible catheter reached approximately the middle (one sow) or the anterior third (five sows) of a uterine horn and was located $25.54 \pm 6.67 \mathrm{~cm}$ (range $8-55 \mathrm{~cm}$ ) from the uterotubal junction. In all cases, the uterine horn adapted to the flexible catheter and formed a spiral shape (Fig. 3).

Six sows were subjected to laparotomy 2 days after deep intrauterine insemination and embryos were collected from 
five sows. These sows had a mean of $22.43 \pm 1.63$ corpora lutea. The recovery rate of embryos and oocytes in relation to the number of corpora lutea was $81.2 \%$. In all of these sows, high proportions of four- to eight-cell embryos were collected from the tip of both uterine horns and no significant differences in the yield of embryos between horns were observed, even when the highest percentages of normal embryos obtained from each uterine horn from each sow were classified as the same horn (uterine horn 1 ; Table 2).

\section{Discussion}

The complexity of the cervical folds and the uterine horn of the pig has discouraged attempts at non-surgical insertion of a catheter into the uterine horn. However, transcervical catheterization of one uterine horn has been reported in non-sedated sows by using a fibreoptic endoscope (Vazquez et al., 1999). This procedure can be performed safely and quickly, making it possible to reach the depth of one uterine horn in about $97 \%$ of the sows.

As it is not necessary to pass an optic system through the cervix or to gain entry into the uterine horn, the new flexible catheter used in the present study was made on the basis of the propulsion force and flexibility of the endoscope used in the previous studies (Vazquez et al., 1999; Martinez et al., 2000). Results from the present study confirm previous findings using endoscopic techniques and demonstrate that the flexible catheter can be inserted in $95.4 \%$ of sows in $<4$ min. However, medium or high difficulties were encountered in inserting the flexible catheter in about $8 \%$ of the sows. In another $5 \%$ of sows, it was not possible to pass the flexible catheter through the cervix. As neither parity number nor duration of lactation were implicated in the different grades of difficulty encountered to pass the cervix, an individual factor could be the main reason for this variability. Sedation of the sows was not necessary during the insertion of the flexible catheter because the procedure was well tolerated by the animals even when the difficulty grade was high or impossible, as observed when using an endoscope (Vazquez et al., 1999). In fact, the behaviour of the sows during insertion of the flexible catheter was similar to the behaviour of the sows during traditional $\mathrm{Al}$, indicating that the welfare of the sows is not perturbed by the deep intrauterine procedure. However, a minimum period of training would be necessary to use this technique on a commercial farm.

After insemination, uterine infections in the form of vaginal discharges were observed in $<1 \%$ of sows inseminated with flexible catheter or traditional Al catheter despite the fact that the flexible catheters were not sterilized between inseminations. This finding indicates that the potential risk of inducing uterine infection during the deep intrauterine procedure is very low and not different from that produced after insertion of a standard intracervical Al spirette, probably because the uterine defence mechanisms are very effective in sows during oestrus (De Winter et al.,
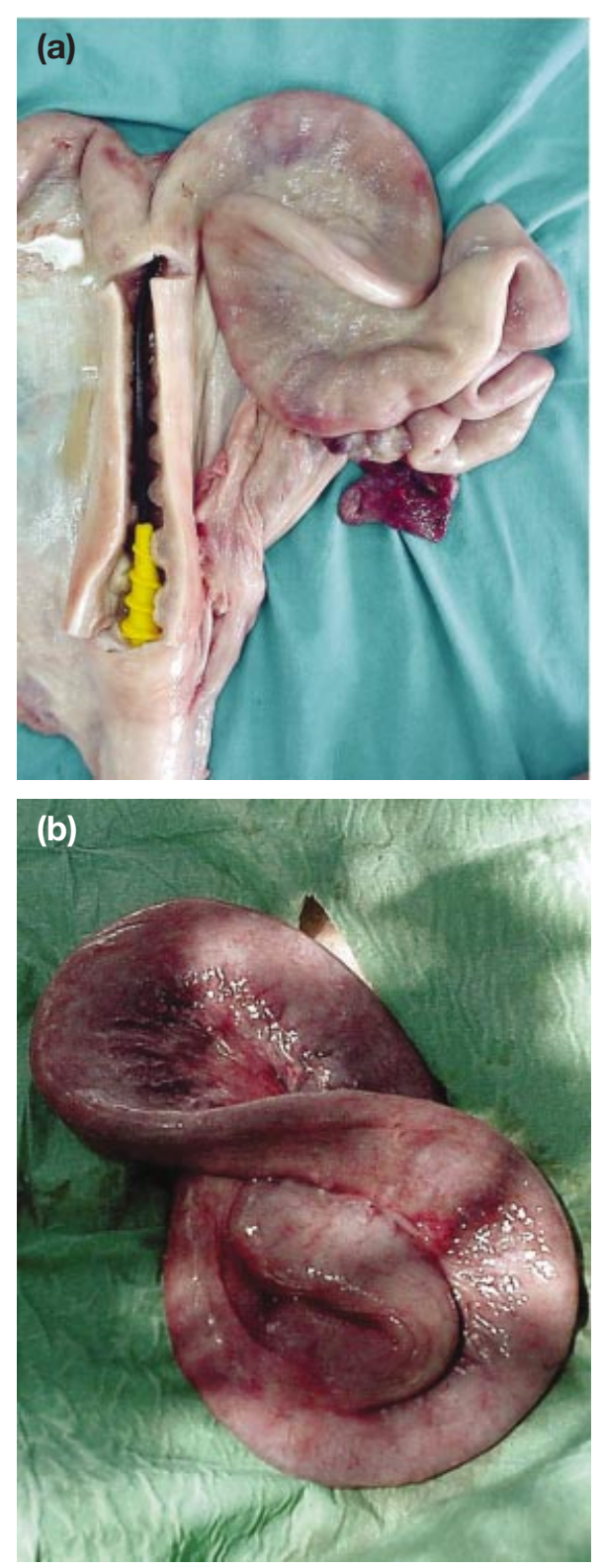

Fig. 3. (a) Silhouette of the flexible catheter inserted into a uterine horn. Reproductive tract was collected from a sow at a local abbatoir. (b) Image obtained during a laparotomy.

1996). The uterine damage after deep intrauterine insemination was not evaluated in the present study. However, the results obtained after deep intrauterine insemination indicate that the procedure does not have a detrimental effect on the fertility of hormonally treated sows inseminated with 5-15 $\times 10^{7}$ spermatozoa. Therefore, it might be considered that the risk of uterine damage during the insertion of the flexible catheter is kept to a minimum.

The fertility results obtained in the present study using deep intrauterine insemination confirm findings using endoscopic techniques (Martinez et al., 2000) and indicate that pregnancy and farrowing rates and litter size obtained 
Table 2. Percentages of normal embryos collected in each uterine horn on day 4 of the oestrous cycle (day $0=$ onset of oestrus) from sows inseminated in one uterine horn with $15 \times 10^{7}$ spermatozoa at $36 \mathrm{~h}$ after hCG treatment using the flexible catheter ${ }^{\mathrm{a}}$

\begin{tabular}{|c|c|c|c|c|c|c|}
\hline \multirow[b]{2}{*}{ Sow } & \multicolumn{3}{|c|}{ Uterine horn 1} & \multicolumn{3}{|c|}{ Uterine horn 2} \\
\hline & $\begin{array}{l}\text { Number of } \\
\text { unfertilized } \\
\text { oocytes/ } \\
\text { degenerated } \\
\text { embryos }\end{array}$ & $\begin{array}{c}\text { Number of } \\
\text { normal } \\
\text { embryos }\end{array}$ & $\begin{array}{c}\text { Normal } \\
\text { embryos }(\%)\end{array}$ & $\begin{array}{l}\text { Number of } \\
\text { unfertilized } \\
\text { oocytes/ } \\
\text { degenerated } \\
\text { embryos }\end{array}$ & $\begin{array}{c}\text { Number of } \\
\text { normal } \\
\text { embryos }\end{array}$ & $\begin{array}{c}\text { Normal } \\
\text { embryos }(\%)\end{array}$ \\
\hline 1 & 0 & 4 & 100.0 & 1 & 8 & 88.9 \\
\hline 2 & 0 & 14 & 100.0 & 0 & 9 & 100.0 \\
\hline 3 & 0 & 12 & 100.0 & 0 & 4 & 100.0 \\
\hline 4 & 0 & 10 & 100.0 & 0 & 7 & 100.0 \\
\hline 5 & 1 & 14 & 93.3 & 2 & 5 & 71.4 \\
\hline Total & 1 & 54 & 98.2 & 3 & 33 & 91.7 \\
\hline
\end{tabular}

aThe uterine horns with the highest percentages of normal embryos (or the highest number of embryos when the proportion was the same) were classified in uterine horn 1 to obtain the maximum difference possible in this parameter between the ipsilateral and contralateral uterine horns in relation to the unknown place of deposition of semen.

when inseminating $5 \times 10^{7}$ spermatozoa in $10 \mathrm{ml}$ per sow were not significantly different from those achieved after standard Al. However, as the number of spermatozoa was decreased to 2.5 and $1.0 \times 10^{7}$ per insemination a decrease in the pregnancy and farrowing rates was observed as compared with the control group (sows traditionally inseminated). Nevertheless, the pregnancy and farrowing rates of sows inseminated with $5 \times 10^{7}$ spermatozoa showed a tendency to be lower (about 9 and $6 \%$, respectively) than those in sows inseminated with $15 \times 10^{7}$ spermatozoa or after standard Al. However, in a study using endoscopy, high farrowing rates were achieved when $5 \times 10^{7}$ spermatozoa was used $(92.3 \%)$, possibly reflecting the small number of sows inseminated ( $n=13$; Martinez et al., 2000). The small number of sows inseminated may explain the discrepancy in relation to the results of the present study, in which 126 sows were inseminated with $5 \times 10^{7}$ spermatozoa. These findings indicate that the number of spermatozoa required to achieve optimal fertility using non-surgical deep intrauterine technique is probably $>5 \times 10^{7}$ and $\leqslant 15 \times 10^{7}$.

The mechanisms by which the number of spermatozoa per insemination can be reduced during insemination in the depth of the uterine horn are not clear. Billions of spermatozoa in a large volume of liquid are deposited intracervically during natural mating or artificial insemination. However, only a very small proportion of the spermatozoa reach the sperm reservoir (Mburu et al., 1996), which is located in the caudal $1-2 \mathrm{~cm}$ of the isthmus (Hunter, 1981, 1984). Here the cells are stored without losing their ability to fertilize (Suarez et al., 1991) and are released just before ovulation (Hunter, 1984). Spermatozoa are eliminated from the uterus by back flow of semen (Steverink et al., 1998) and by local phagocytosis (Rozeboom et al., 1998, 1999). These processes determine that only about $10 \%$ of the spermatozoa inseminated can be recovered from the uterus $1-2 \mathrm{~h}$ after semen deposition (First et al., 1968; Viring, 1980). However, some spermatozoa reach the sperm reservoir within 15 min after insemination (First et al., 1968) and an adequate number of spermatozoa to ensure optimal fertilization are present in the sperm reservoir within $1-2 \mathrm{~h}$ of mating (Hunter, 1981, 1984). Hunter (1982) proposed that although uterine contractions should assist sperm transport, the large volume of semen deposited during natural mating or $\mathrm{Al}$ could redistribute a portion of the semen to the region of the uterotubal junction, which is bathed in a sperm suspension by the completion of mating (Hunter, 1982) or Al (Baker and Degen, 1972). The deposition of a small number of spermatozoa in a small volume of medium close to the uterotubal junction may be similar to an aliquot of the ejaculate or of a standard $\mathrm{Al}$ dose reaching the uterotubal junction during service or $\mathrm{Al}$, as has been suggested in horses (Morris et al., 2000). However, results from a study by Martinez et al. (2000) indicate that it is not necessary to deposit semen in a precise region of the uterotubal junction to obtain adequate farrowing rates and litter size by inseminating a small number of spermatozoa in a small volume of medium. In the present study, $5 \times 10^{7}$ spermatozoa were deposited near the middle of one uterine horn. These conditions were sufficient to enable formation of a sperm reservoir in the caudal isthmus of at least one oviduct, because no significant differences were observed in farrowing rates from those obtained after standard $\mathrm{Al}\left(3 \times 10^{9}\right.$ spermatozoa in $100 \mathrm{ml}$ in two inseminations per sow). In the present study, despite the fact that deep intrauterine insemination was performed closer to the uterotubal junction (anterior third of the one uterine horn), the number of spermatozoa inseminated cannot be $<5 \times 10^{7}$ without a reduction in fertility.

In addition to dose volume and deposition of the spermatozoa close to uterotubal junction, other factors could be implicated in the differences in requirements between intra- 
cervical and deep intrauterine insemination procedures. There are two basic differences between these two procedures. Firstly, during intracervical insemination spermatozoa come into contact with the cervical canal and its secretions, which do not occur during deep intrauterine insemination. As the traditional deposition of semen in pigs has generally been considered 'intrauterine', little attention has been given to the sperm-cervical mucus interaction in this species. Secondly, deep intrauterine insemination produces a large distension of the cervix and uterine horn, which might induce a greater release of hormones implicated in uterine contractability and sperm transport compared with the traditional insemination method. Investigations are currently in progress to clarify some of these speculations.

The litter sizes obtained in the present study after a single insemination in the depth of one uterine horn were not significantly different from those of the standard inseminated control group. It is possible that the hormonal treatment of sows inseminated in the depth of one uterine horn increased the number of ovulations in each ovary and, as a result a greater number of oocytes could be fertilized in the oviduct ipsilateral to the uterine horn inseminated. This finding could explain the large number of piglets born under the experimental conditions used in the present study if fertilization had occurred in only one oviduct. However, this hypothesis is not consistent for two principal reasons. Firstly, the number of corpora lutea counted in the sows subjected to laparotomy was about 22 , which indicates that the treatment was not effective in inducing superovulation. Secondly, the proportion of embryos collected after deep intrauterine insemination with $15 \times 10^{7}$ spermatozoa was similar in both uterine horns. This finding clearly demonstrates that when spermatozoa are deposited close to the uterotubal junction in only one uterine horn, spermatozoa are able to reach the contralateral oviduct and fertilize the oocytes. In sows with one uterine horn sectioned via laparoscopy it was possible to confirm that spermatozoa are able to reach the contralateral oviduct and fertilize a large proportion of the oocytes (92\%) following a transperitoneal pathway (unpublished data). This finding is not unexpected, as sperm transport and fertilization have been reported after intraperitoneal deposition of boar semen (Hunter, 1978). Furthermore, it has been suggested that spermatozoa pass through the oviduct into the abdominal cavity during the first hours after intracervical deposition of spermatozoa (Viring and Einarsson, 1981). Whether spermatozoa can also reach the contralateral oviduct via the uterus after deep intrauterine insemination is currently under investigation.

In conclusion, a new technique for non-surgical deep uterine insemination in pigs has been developed. With this technique, it is possible to pass the cervical canal and reach the depth of one uterine horn in about $95 \%$ of the sows in $<4$ min. In comparison with standard $\mathrm{Al}\left(3 \times 10^{9}\right.$ spermatozoa in $80-100 \mathrm{ml}$ ), a $20-60$-fold reduction in the number of spermatozoa inseminated and at least an 8-10- fold reduction in the dose volume can be used for $\mathrm{Al}$ without a decrease in fertility.

Deep intrauterine insemination might be of great benefit to the pig industry because the number of seminal doses per year obtained from a boar could be increased from the current recommended 2000 to at least 40000 . Other biotechnologies, such as frozen semen, flow-sorted spermatozoa or non-surgical embryo transfer could be enhanced by the deep intrauterine insemination technique. In addition, studies of the biological mechanisms involved in sperm transport and possibly sperm selection by the female reproductive tract could be undertaken with heterospermic inseminations and accurate semen delivery to various depths of the female reproductive tract.

The authors are grateful to $\mathrm{H}$. Mesa for statistical assistance. Financial support from FEDER (1FD97-370), CDTI B288/98, MEC (PR2000-179) and Miller Fund-MU is also most gratefully acknowledged.

\section{References}

Baker RD and Degen AA (1972) Transport of live and dead boar spermatozoa within the reproductive tract of gilts Journal of Reproduction and Fertility 28 369-377

Baker RD, Dziuk PJ and Norton HW (1968) Effect of volume of semen, number of sperm and drugs on transport of sperm in artificially inseminated gilts Journal of Animal Science 27 88-93

Day BN (1979) Embryo transfer in swine Theriogenology 11 27-31

De Winter PJJ, Verdonck M, De Kruif A, Coryn M, Deluyker HA, Devriese LA and Haesebrouck $\mathbf{F}$ (1996) The relationship between the blood progesterone concentration at early metoestrus and uterine infection in the sow Animal Reproduction Science 41 51-59

Dziuk PJ and Henshaw G (1958) Fertility of boar semen artificially inseminated following in vitro storage Journal of Animal Science 17 554-558

First NL, Short RE, Peters JB and Stratman FW (1968) Transport and loss of boar spermatozoa in the reproductive tract of the sow Journal of Animal Science 27 1037-1040

Hagen DR, Prather RS, Sims MM and First NL (1991) Development of onecell porcine embryos to the blastocyst stage in simple media Journal of Animal Science 69 1147-1150

Hunter RHF (1978) Intraperitoneal insemination, sperm transport and capacitation in the pig Animal Reproduction Science 1 167-179

Hunter RHF (1981) Sperm transport and reservoirs in the pig oviduct in relation to the time of ovulation Journal of Reproduction and Fertility $\mathbf{6 3}$ 109-117

Hunter RHF (1982) Interrelationships between spermatozoa, the female reproductive tract and the egg investments. In Control of Pig Reproduction pp 585-601 Eds DJA Cole and GR Foxcroft. Butterworth, London

Hunter RHF (1984) Pre-ovulatory arrest and peri-ovulatory redistribution of competent spermatozoa in the isthmus of the pig oviduct Journal of Reproduction and Fertility $\mathbf{7 2}$ 203-211

Krueger C and Rath D (2000) Intrauterine insemination in sows with reduced sperm number Reproduction, Fertility and Development 12 $113-117$

Krueger C, Rath D and Johnson LA (1999) Low dose insemination in synchronized gilts Theriogenology 52 1363-1373

Martinez EA, Vazquez JM, Vazquez JL, Lucas X, Gil MA, Parrilla I and Roca J (2000) Successful low-dose insemination by a fiberoptic endoscope technique Theriogenology 53201 (Abstract)

Mburu JN, Einarsson S, Lundeheim N and Rodriguez-Martinez H (1996) Distribution, number and membrane integrity of spermatozoa in the pig oviduct in relation to spontaneous ovulation Animal Reproduction Science 45 109-121 
Morris LHA, Hunter RHF and Allen WR (2000) Hysteroscopic insemination of small numbers of spermatozoa at the uterotubal junction of preovulatory mares Journal of Reproduction and Fertility 118 95-100

Polge C (1956) Artificial insemination in pigs The Veterinary Record $\mathbf{6 8}$ $62-76$

Pursel VG and Johnson LA (1975) Freezing of boar spermatozoa: fertilizing capacity with concentrated semen and a new thawing procedure Journal of Animal Science 40 99-102

Rozeboom KJ, Troedsson MHT and Crabo BG (1998) Characterization of uterine leukocyte infiltration in gilts after artificial insemination Journal of Reproduction and Fertility 114 195-199

Rozeboom KJ, Troedsson MHT, Molitor TW and Crabo BG (1999) The effect of spermatozoa and seminal plasma on leukocyte migration into the uterus of gilts Journal of Animal Science 77 2201-2206

SAS/STAT (1999) Version 8 Users' Guide SAS Institute, Cary, NC

Steverink DWB, Soede NM, Bouwman EG and Kemp B (1998) Semen backflow after insemination and its effect on fertilization results in sows Animal Reproduction Science 54 109-119

Stratman FW and Self HL (1968) Effect of semen volume and number of sperm on fertility and embryo survival in artificially inseminated gilts Journal of Animal Science 19 1081-1088

Suarez S, Redfern K, Raynor P, Martin F and Phillips DM (1991) Attachment of boar sperm to mucosal explants of oviduct in vitro: possible role in formation of a sperm reservoir Biology of Reproduction 44 998-1004

Vazquez JL, Martinez EA, Vazquez JM, Lucas X, Gil MA, Parrilla I and Roca J (1999) Development of a non-surgical deep intrauterine insemination technique. In IV International Conference on Boar Semen Preservation p 35 Beltsville, MD

Viring S (1980) Distribution of live and dead spermatozoa in the genital tract of gilts at different times after insemination Acta Veterinaria Scandinavica 21 145-149

Viring S and Einarsson S (1981) Sperm distribution within the genital tract of naturally inseminated gilts Nordiska Veterinarian Medicine 33 145-149

Weitze KF (2000) Update on the worldwide application of swine AI. In Boa Semen Preservation IV pp 141-145 Eds LA Johnson and HD Guthrie. Allen Press Inc., Lawrence, KS

Wiggins EL, Grummer RH and Casida LE (1951) Minimal volume of semen and number of sperm for fertility in artificial insemination in swine Journal of Animal Science 10 138-143

Received 23 July 2001.

First decision 21 August 2001.

Accepted 10 October 2001. 